\title{
DNA specificity in two Hedysarum sections, Hedysarum and Multicaulia (Siberia, Russia) inferred from ETS sequence data
}

\author{
Natalia Nuzhdina ${ }^{1, *}$, Abdollah Ghorttapeh Hassanzadeh ${ }^{2}$ and Olga Dorogina ${ }^{1}$ \\ ${ }^{1}$ Central Siberian Botanical Garden of the Siberian Brunch of the Russian Academy of Sciences, \\ Novosibirsk, Russia \\ ${ }^{2}$ Horticulture Crop Science Research Department, West Azerbaijan Agricultural and Natural \\ Resources Research and Education Center (AREEO), Urmia, Iran
}

\begin{abstract}
Based on sequence data of the nuclear ETS markers of 20 species, the relationships of sections Hedysarum and Multicaulia were assessed. Phylogenetic analysis was performed on the complete sequences using the Neighbor-Joining methods. The nuclear marker ETS is investigated for the first time in the most species excluding $H$. hedysaroides, $H$. inundatum, $H$. ferganense, and $H$. neglectum. The length of sequences varies from 349 to 357 nucleotides. It is noteworthy that ETS data significantly discriminate $H$. ferganense from both sections, Hedysarum and Multicaulia. Moreover, our results did not support the subsectional division of Multicaulia section. Therefore, the ETS sequence data obtained in our study for 20 taxa of Hedysarum evidence the nonmonophyly of section Multicaulia.
\end{abstract}

\section{Introduction}

The genus Hedysarum L. (sweet vetch) is the largest of the tribe Hedysareae DC., the legume family (Fabaceae L.) and includes up to 200 species of perennial herbs $[1,2]$. Hedysarum species have been successfully used due to their crop and fertilization importance and as ornamental, melliferous, and medical plants [3].

The monophyletic origin of the genus Hedysarum and tribe Hedysareae has been discussed [1, 4]. Last decades, nuclear and plastid DNA markers provided evidences concerned with molecular divergence and phylogenetic relationship in plants. The Hedysarum genus were re-defined using both nuclear and chloroplast DNA sequences [1]. The latest infrageneric system was performed based on 58 species Hedysareae using five nuclear and five chloroplast markers [1]. It was demonstrated by Mironov [5] that pericarp anatomy has a section-specific properties for Hedysarum (previously - Obscura, or Gamotion) and Multicaulia sections. Choi and Ohashi [4] believed that sweet vetch species belonging to these sections are characterized by a different chromosome number, $\mathrm{x}=7$ and $\mathrm{x}=8$, correspondingly.

\footnotetext{
* Corresponding author: nsnuzhdina@gmail.com
} 
It was demonstrated that the nuclear DNA marker, external transcribed spacer (ETS) region of the $18 \mathrm{~S}$ rRNA gene is a useful tool for studying the phylogeny of closely related species. Up to now, previous ETS molecular dating efforts used limited Hedysarum taxa sampling. Thus, we want to achieve the following objectives: (1) to access a molecular variability in Hedysarum species by sequencing ETS region of a nuclear DNA; (2) to resolve the relationships among the species of two sections of a sweet vetch, Hedysarum and Multicaulia.

\section{Materials and methods}

The present study was based on leaf material collected by the first author in 2010 and 2011, and the materials removed from herbarium specimens preserved in NSK herbarium (Novosibirsk, Russia). The samples analyzed in this study were collected from Siberia, Russia excluding $H$. hedysaroides (Italy), H. ferganense (Tajikistan) and H. truncatum (Kamchatka, Russian Far East).

The analysis included a total of 21 samples representing two sections of Hedysarum. DNA was purified from $40 \mathrm{mg}$ of foliar issue using the NucleoSpin Plant II Kit (MachereyNagel, USA) according to the manufacturer's protocol. The DNA concentration and purity was determined using a BioSpectrometer kinetic and $\mu$ Cuvette G 1.0 (Eppendorf, Germany). Corethrodendron fruticosum $(=H$. fruticosum) was used as outgroup.

Genomic DNA was amplified for ETS, using ETS-Hedy (F) and 18S-IGS (R) primers and the protocol (Liu et al. 2017). All PCR reactions were done in a Thermal Cycler T1000 (Bio-Rad, USA). Amplified DNA fragments were stained with SYBR-Green (ThermoFischer Scientific, USA) and visualized in $1.5 \%$ agarose gel using Gel-Doc XR+ documentation system equipped with the ImageLab Software (Bio-Rad, USA).

PCR fragments obtained were then purified by sorption on AMPureXP magnetic particles (Agencourt, USA) and sequenced directly using the BigDye Ready Reaction DNA Sequencing Kit v. 3.1 (Thermo Fischer Scientific, USA). The Sanger reaction products were purified from unincorporated fluorescent dyes by centrifugation (900 $g, 2 \mathrm{~min}$ ) through a column packed with $750 \mu \mathrm{L}$ of the Sephadex G-50 Fine suspension (GE Healthcare, USA) and analyzed on 3130XL automatic genetic analyzer (Applied Biosystems, USA) in the SB RAS Genomics Core Facility (ICBFM, Novosibirsk).

The nucleotide sequences were aligned by Clustal-W algorithm and the NeighborJoining trees were computed using the program MEGA 10.0.5. Robustness of the brunches was calculated using 1000 bootstrap replications (BS).

\section{Results and discussion}

Based on the sequence data of ETS the genetic relationship between two sections of a sweet vetch, Hedysarum and Multicaulia were assessed (Fig. 1). The length of sequences varied from 349 (H. consanguineum) to 357 nucleotides (H. dahuricum, H. dasycarpum, $H$. ferganense). The molecular data of nuclear ETS marker used were polymorphic and provided substitutions to delimit the most species.

The nuclear ETS marker was investigated for the first time in the most taxa excluding $H$. hedysaroides, $H$. inundatum, $H$. ferganense, and $H$. neglectum. There were no indels among several species from Hedysarum section, H. consanguineum, $H$. arcticum, and $H$. inundatum, and between $H$. alpinum - $H$. vicioides. 


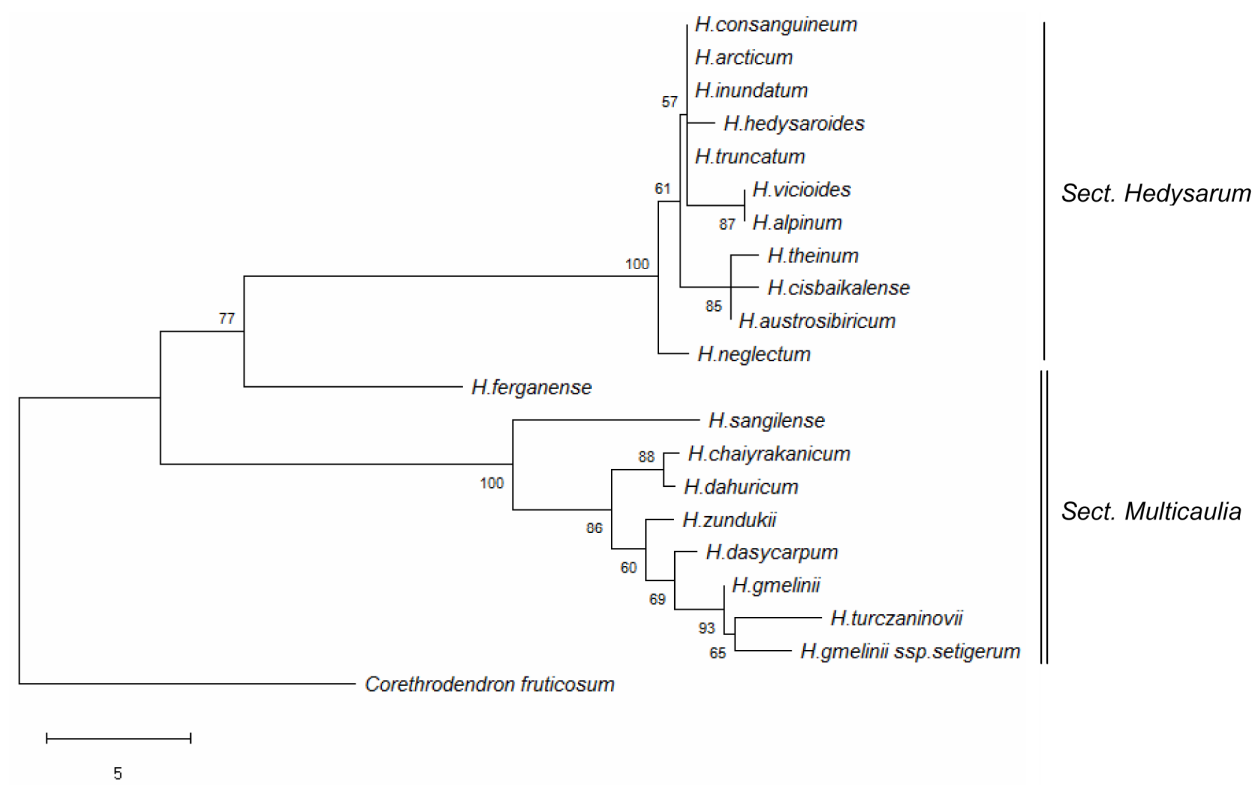

Fig. 1. Phylogenetic relationships among Hedysarum species based on Neighbor-Joining method using ETS sequence data. Bootstrap percentages are shown next to the branches.

The nuclear ETS marker allowed us to clarify relationships between two sections, Hedysarum and Multicaulia with 20 sampled accessions. Results of DNA sequencing presented herein demonstrated that the relationship registered between two sections using ETS sequences was consistent with the trnL-F chloroplast data [6].

According to the Neighbour-Joining tree based on ETS sequencing, Hedysarum species belonging to different sections, Hedysarum and Multicaulia, were grouped separately (100 BS). The exception was $H$. ferganense which had an intermediate position between the section; the bootstrap support was of $77 \%$. The separate clade with the single species, mountain legume $H$. ferganense, usual in Altai and Middle Asia, is placed closer to Hedysarum section clade rather than Multicaulia which the species belongs to.

Among Multicaulia section, there was a group of closely related species: two endemics, $H$. turczaninovii and $H$. gmelinii subsp. setigerum, and its widespread congener H. gmelinii. Hence, the molecular DNA similarity between $H$. gmelinii and its stemless subspecies evidenced from trnL-F sequence data previously [6] has been improved. Another two local endemics, $H$. chaiyrakanicum and $H$. dahuricum possessed a high molecular similarity according their ETS nucleotide sequences (88 BS). Both species possess non-typical pale yellow or pinkish flowers and are distributed among steppe vegetation strictly, on stony slopes and rocks.

Our results revealed incongruence between ETS-tree location and subsectional affinities among Multicaulia section taxa. Species belonging to Subacaulia and Multicaulia subsections [4] were dispersed chaotically which has evidenced the unnatural subdivision of Multicaulia section, whereas the sequences of trnL-F locus of cpDNA analyzed in our previous study [6] have discovered the heterogeneity (biphyly) of section Hedysarum. Taking into consideration the intermediate position of $H$. ferganense at ETS phylogenetic tree, we can assume therefore that our data supports the hypothesis of non-monophyletic origin of Hedysarum proposed by Liu et al. (2017).

This work was supported by the budgetary project of the CSBG "Assessment of morphogenetic potential of Northern Asian plant populations using experimental methods" (no. AAAA-A17-117012610051-5). 


\section{References}

1. P.-L. Liu, J. Wen, L. Duan, E. Arslan, K. Ertugrul, Z.-Y. Chang, PlosOne 12(1) (2017)

2. G. P. Yakovlev, A. K. Sytin, Y. R. Roskov, Legumes of Northern Eurasia: a check-list (Royal Botanical Gardens, Kew, 1996)

3. M. Yu. Vdovichenko, I. N. Kuzovkina, Kh. Petts, B. Shnaider, Fiziol. Rast. 54(4) (2007).

4. B.-H. Choi, H. Ohashi, Taxon, 52 (2003)

5. Ye. M. Mironov, Bull. Mosc. Soc. Natur. Biol. Ser. 105(1) (2009)

6. N. S. Nuzhdina, A. A. Bondar, O. V. Dorogina, Rus. J. Genet. 54(11) (2018) 